\title{
A Simple Generalisation of Kirk's Approximation for Multi-Asset Spread Options by the Lie-Trotter Operator Splitting Method
}

\author{
Chi-Fai Lo \\ Institute of Theoretical Physics and Department of Physics, The Chinese University of Hong Kong, Hong Kong, \\ China \\ Email: cflo@phy.cuhk.edu.hk
}

Received 2 March 2014; revised 3 April 2014; accepted 21 April 2014

Copyright (C) 2014 by author and Scientific Research Publishing Inc. This work is licensed under the Creative Commons Attribution International License (CC BY). http://creativecommons.org/licenses/by/4.0/

\section{(c) (i) Open Access}

\begin{abstract}
In this paper, by means of the Lie-Trotter operator splitting method, we have presented a new unified approach not only to rigorously derive Kirk's approximation but also to obtain a generalisation for multi-asset spread options in a straightforward manner. The derived price formula for the multi-asset spread option bears a great resemblance to Kirk's approximation in the two-asset case. More importantly, our approach is able to provide a new perspective on Kirk's approximation and the generalization; that is, they are simply equivalent to the Lie-Trotter operator splitting approximation to the Black-Scholes equation.
\end{abstract}

\section{Keywords}

Lognormal Random Variables, Black-Scholes Equation, Spread Options, Kirk's Approximation, Lie-Trotter Operator Splitting Method

\section{Introduction}

Spread options, whose payoff is contingent upon the price difference of two underlying assets form the simplest type of multi-asset options, are very popular in markets as diverse as interest rate markets, currency and foreign exchange markets, commodity markets, and energy markets nowadays [1]. Despite the rapid development of spread options, pricing spread options is a very challenging task and receives much attention in the literature. The major difficulty lies in the lack of knowledge about the distribution of the spread of two correlated 
lognormal random variables. The simplest approach is to evaluate the expectation of the final payoff over the joint probability distribution of the two correlated lognormal underlyings by means of numerical integration. However, practitioners often prefer to use analytical approximations rather than numerical methods because of their computational ease. Among various analytical approximations, e.g. Carmona and Durrleman [1], Deng et al. [2], Bjerksund and Stensland [3], Venkatramana and Alexander [4], Kirk's approximation seems to be the most widely used and is the current market standard, especially in the energy markets [5]. It is well known that Kirk's approximation extends from Margrabe's exchange option formula with no rigorous derivation [6]. Recently, Lo [7] applied the idea of WKB method to provide a derivation of Kirk's approximation and discuss its validity. Nevertheless, it is not straightforward to provide a generalisation of Kirk's approximation for the case of multi-asset spread option via this approach.

Accordingly, it is the aim of this paper to present a simple unified approach, namely the Lie-Trotter operator splitting method [8], not only to rigorously derive Kirk's approximation but also to obtain a generalisation for the case of multi-asset spread option in a straightforward manner. The derived price formula for the multi-asset spread option bears a great resemblance to Kirk's approximation in the two-asset case. More importantly, the proposed approach is able to provide a new perspective on Kirk's approximation and the generalisation; that is, they are simply equivalent to the Lie-Trotter operator splitting approximation to the Black-Scholes equation. Illustrative numerical examples for the three-asset spread options are also shown to demonstrate both the accuracy and efficiency of the extended Kirk approximation. Furthermore, it should be emphasized that our approach is completely different from the extended Kirk approximation proposed by $\mathrm{Li}$ et al. [9]. Li et al. suggested to approximate the sum of $N-1$ lognormal assets as a single lognormal variable and then apply Kirk's approximation directly to this single lognormal variable and the remaining asset to price the $N$-asset spread option.

\section{Two-Asset Spread Options}

The price of a European call spread option obeys the two-dimensional Black-Scholes equation

$$
\left\{\frac{1}{2} \sigma_{1}^{2} F_{1}^{2} \frac{\partial^{2}}{\partial F_{1}^{2}}+\rho \sigma_{1} \sigma_{2} F_{1} F_{2} \frac{\partial^{2}}{\partial F_{1} \partial F_{2}}+\frac{1}{2} \sigma_{2}^{2} F_{2}^{2} \frac{\partial^{2}}{\partial F_{2}^{2}}-r-\frac{\partial}{\partial \tau}\right\} P\left(F_{1}, F_{2}, \tau\right)=0
$$

with the final payoff condition

$$
P\left(F_{1}, F_{2}, 0\right)=\max \left(F_{1}-F_{2}-K, 0\right) .
$$

Here $F_{1}$ and $F_{2}$ are the future prices of the two lognormal underlying assets, $\sigma_{1}$ and $\sigma_{2}$ are the volatilities, $\rho$ is the correlation between the two assets, $K$ is the strike price, $r$ is the risk-free interest rate, and $\tau$ denotes the time-to-maturity. It is well known that no analytical solution is available in closed form and one needs to resort to numerical methods. In the following, we propose a simple derivation of the well-known price formula of Kirk’s approximation by means of the Lie-Trotter operator splitting method [8].

\section{Proposition 1:}

The price of the two-asset spread option can be approximated by

$$
P\left(F_{1}, F_{2}, \tau\right) \approx \mathrm{e}^{-r \tau}\left\{F_{1} N\left(\xi_{1}\right)-\left(F_{2}+K\right) N\left(\xi_{2}\right)\right\}
$$

where

$$
\begin{gathered}
\xi_{1}=\frac{1}{\tilde{\sigma}_{-} \sqrt{\tau}}\left\{\ln \left(\frac{F_{1}}{F_{2}+K}\right)+\frac{1}{2} \tilde{\sigma}_{-}^{2} \tau\right\} \\
\xi_{2}=\xi_{1}-\tilde{\sigma}_{-} \sqrt{\tau} \\
\tilde{\sigma}_{-}=\sqrt{\sigma_{1}^{2}-2 \rho \sigma_{1} \tilde{\sigma}_{2}+\tilde{\sigma}_{2}^{2}} \\
\tilde{\sigma}_{2}=\sigma_{2}\left(\frac{F_{2}}{F_{2}+K}\right) .
\end{gathered}
$$




\section{Proof:}

In terms of the two new variables

$$
R_{1}=\frac{F_{1}}{F_{2}+K} \text { and } R_{2}=F_{2}+K,
$$

Equation (1) can be rewritten as follows:

$$
\left\{\hat{L}_{0}+\hat{L}_{1}-r-\frac{\partial}{\partial \tau}\right\} P\left(R_{1}, R_{2}, \tau\right)=0,
$$

where

$$
\begin{gathered}
\hat{L}_{0}=\frac{1}{2} \tilde{\sigma}_{-}^{2} R_{1}^{2} \frac{\partial^{2}}{\partial R_{1}^{2}} \\
\hat{L}_{1}=\frac{1}{2} \tilde{\sigma}_{2}^{2} R_{2}^{2} \frac{\partial^{2}}{\partial R_{2}^{2}}+\left(\rho \sigma_{1}-\tilde{\sigma}_{2}\right) \tilde{\sigma}_{2} R_{1} R_{2} \frac{\partial^{2}}{\partial R_{1} \partial R_{2}}-\left(\rho \sigma_{1}-\tilde{\sigma}_{2}\right) \tilde{\sigma}_{2} R_{1} \frac{\partial}{\partial R_{1}} .
\end{gathered}
$$

The final payoff condition now becomes

$$
P\left(R_{1}, R_{2}, 0\right)=R_{2} \max \left(R_{1}-1,0\right) .
$$

Accordingly, the formal solution of Equation (9) is given by

$$
P\left(R_{1}, R_{2}, \tau\right)=\mathrm{e}^{-r \tau} \exp \left\{\tau\left(\hat{L}_{0}+\hat{L}_{1}\right)\right\} R_{2} \max \left(R_{1}-1,0\right) .
$$

Since the exponential operator $\exp \left\{\tau\left(\hat{L}_{0}+\hat{L}_{1}\right)\right\}$ is difficult to evaluate, the Lie-Trotter operator splitting method [8] can be applied to approximate the operator by (see the Appendix)

$$
\hat{O}^{L T}=\exp \left\{\tau \hat{L}_{0}\right\} \exp \left\{\tau \hat{L}_{1}\right\}
$$

and obtain an approximation to the formal solution $P\left(F_{1}, F_{2}, \tau\right)$, namely

$$
P^{L T}\left(R_{1}, R_{2}, \tau\right)=\hat{O}^{L T} R_{2} \max \left(R_{1}-1,0\right)=R_{2} \exp \left\{\tau \hat{L}_{0}\right\} \max \left(R_{1}-1,0\right) \equiv R_{2} C\left(R_{1}, \tau\right),
$$

for

$$
\hat{L}_{1} R_{2} \max \left(R_{1}-1,0\right)=0 \Rightarrow \exp \left\{\tau \hat{L}_{1}\right\} R_{2} \max \left(R_{1}-1,0\right)=R_{2} \max \left(R_{1}-1,0\right) .
$$

It is not difficult to recognise that $C\left(R_{1}, \tau\right)$ satisfies the partial differential equation

$$
\left\{\frac{1}{2} \tilde{\sigma}_{-}^{2} R_{1}^{2} \frac{\partial^{2}}{\partial R_{1}^{2}}-r-\frac{\partial}{\partial \tau}\right\} C\left(R_{1}, \tau\right)=0
$$

with the initial condition: $C\left(R_{1}, 0\right)=\max \left(R_{1}-1,0\right)$, and that the admissible solution is given by

$$
C\left(R_{1}, \tau\right)=\mathrm{e}^{-r \tau}\left\{R_{1} N\left(\xi_{1}\right)-N\left(\xi_{2}\right)\right\},
$$

where $N(\cdot)$ is the cumulative normal distribution function and

$$
\begin{gathered}
\xi_{1}=\frac{1}{\tilde{\sigma}_{-} \sqrt{\tau}}\left\{\ln R_{1}+\frac{1}{2} \tilde{\sigma}_{-}^{2} \tau\right\} \\
\xi_{2}=\xi_{1}-\tilde{\sigma}_{-} \sqrt{\tau} .
\end{gathered}
$$

As a result, we obtain

$$
P^{L T}\left(R_{1}, R_{2}, \tau\right)=\mathrm{e}^{-r \tau} R_{2}\left\{R_{1} N\left(\xi_{1}\right)-N\left(\xi_{2}\right)\right\}
$$

which is exactly the approximate price formula given in Equation (3). (Q.E.D.)

In terms of the spot asset prices, namely $S_{i} \equiv F_{i} \exp (-r \tau)$, the price formula given in Equation (3) turns out 
to be identical to the price formula of Kirk's approximation

$$
P_{\text {Kirk }}\left(S_{1}, S_{2}, \tau\right)=S_{1} N\left(d_{1}\right)-\left(S_{2}+K \mathrm{e}^{-r \tau}\right) N\left(d_{2}\right)
$$

where

$$
\begin{gathered}
d_{1}=\frac{\ln S_{1}-\ln \left(S_{2}+K \mathrm{e}^{-r \tau}\right)}{\bar{\sigma}_{-} \sqrt{\tau}}+\frac{1}{2} \bar{\sigma}_{-} \sqrt{\tau} \\
d_{2}=d_{1}-\bar{\sigma}_{-} \sqrt{\tau} \\
\bar{\sigma}_{-}=\sqrt{\sigma_{1}^{2}-2 \rho \sigma_{1} \bar{\sigma}_{2}+\bar{\sigma}_{2}^{2}} \\
\bar{\sigma}_{2}=\sigma_{2}\left(\frac{S_{2}}{S_{2}+K \mathrm{e}^{-r \tau}}\right) .
\end{gathered}
$$

It should be noted that for the Lie-Trotter operator splitting approximation to be valid, $\bar{\sigma}_{-}^{2} \tau$ needs to be sufficiently small, namely $\bar{\sigma}_{-}^{2} \tau \ll 1$. In accordance with Equation (25), this implies that Kirk's approximation is more favourable for positive correlation $\rho$ between the two assets. Furthermore, for $K=0$, the operators $\hat{L}_{0}$ and $\hat{L}_{1}$ commute so that the Lie-Trotter splitting approximation becomes exact and Margrabe's formula is recovered.

\section{Multi-Asset Spread Options}

To price a European $N$-asset call spread option, we need to solve the $N$-dimensional Black-Scholes equation

$$
\left\{\sum_{i, j=1}^{N} \frac{1}{2} \rho_{i j} \sigma_{i} \sigma_{j} F_{i} F_{j} \frac{\partial^{2}}{\partial F_{i} \partial F_{j}}-r-\frac{\partial}{\partial \tau}\right\} P\left(F_{1}, F_{2}, \cdots, F_{N}, \tau\right)=0
$$

subject to the final payoff condition

$$
P\left(F_{1}, F_{2}, \cdots, F_{N}, 0\right)=\max \left(F_{N}-\sum_{j=1}^{N-1} F_{j}-K, 0\right),
$$

where $F_{i}$ is the future price of the lognormal underlying asset $i$ with the volatility $\sigma_{i}, \rho_{i j}$ is the correlation between the assets $i$ and $j, K$ is the strike price, $r$ is the risk-free interest rate, and $\tau$ is the timeto-maturity. Unfortunately, this is a very formidable task. In the following, we apply the Lie-Trotter operator splitting method to derive a closed-form approximate price formula for the multi-asset spread option, which bears a great resemblance to the price formula of Kirk’s approximation in the two-asset case.

\section{Proposition 2:}

The price of the $N$-asset spread option can be approximated by

$$
P\left(F_{1}, F_{2}, \cdots, F_{N}, \tau\right) \approx \mathrm{e}^{-r \tau}\left\{F_{N} N\left(\theta_{1}\right)-\left(\sum_{j=1}^{N-1} F_{j}+K\right) N\left(\theta_{2}\right)\right\}
$$

where

$$
\begin{gathered}
\theta_{1}=\frac{1}{\bar{\sigma}_{-} \sqrt{\tau}}\left\{\ln \left(\frac{F_{N}}{\sum_{j=1}^{N-1} F_{j}+K}\right)+\frac{1}{2} \bar{\sigma}_{-}^{2} \tau\right\} \\
\theta_{2}=\theta_{1}-\bar{\sigma}_{-} \sqrt{\tau} \\
\bar{\sigma}_{-}=\sqrt{\sigma_{N}^{2}-2 \tilde{\rho} \sigma_{N} \bar{\sigma}_{+}+\bar{\sigma}_{+}^{2}} \\
\bar{\sigma}_{+}=\tilde{\sigma}_{+}\left(\frac{\sum_{j=1}^{N-1} F_{j}}{\sum_{j=1}^{N-1} F_{j}+K}\right)
\end{gathered}
$$




$$
\begin{gathered}
\tilde{\sigma}_{+}=\frac{\sqrt{\sum_{j, k=1}^{N-1} \rho_{j k} \sigma_{j} \sigma_{k} F_{j} F_{k}}}{\sum_{j=1}^{N-1} F_{j}} \\
\tilde{\rho}=\frac{1}{\tilde{\sigma}_{+}}\left(\frac{\sum_{j=1}^{N-1} \rho_{j N} \sigma_{j} F_{j}}{\sum_{j=1}^{N-1} F_{j}}\right) .
\end{gathered}
$$

\section{Proof:}

Introducing the new variables: $f_{i} \equiv F_{i}-F_{+}$for $i=1,2, \cdots, N-2$ and $F_{+} \equiv \sum_{j=1}^{N-1} F_{j}$, Equation (27) can be cast in the form

$$
\left\{\hat{L}_{N}+\hat{L}_{+}+\hat{L}_{N+}+\hat{L}_{R}-r-\frac{\partial}{\partial \tau}\right\} P\left(F_{1}, F_{2}, \cdots, F_{N}, \tau\right)=0,
$$

where

$$
\begin{gathered}
\hat{L}_{N}=\frac{1}{2} \sigma_{N}^{2} F_{N}^{2} \frac{\partial^{2}}{\partial F_{N}^{2}} \\
\hat{L}_{+}=\frac{1}{2} \tilde{\sigma}_{+}^{2} F_{+}^{2} \frac{\partial^{2}}{\partial F_{+}^{2}} \\
\hat{L}_{N+}=\tilde{\rho} \sigma_{N} \tilde{\sigma}_{+} F_{+} F_{N} \frac{\partial^{2}}{\partial F_{+} \partial F_{N}} \\
\hat{L}_{R}=\frac{1}{2} \sum_{k=1}^{N-2 N-1}\left(\rho_{n=1} \sigma_{k} F_{k}-\sum_{m=1}^{N-1} \rho_{m n} \sigma_{m} F_{m}\right) \sigma_{n} F_{n} \frac{\partial^{2}}{\partial F_{+} \partial f_{k}} \\
+\frac{1}{2} \sum_{k=1}^{N-2} \sum_{n=1}^{N-2}\left\{\left(\rho_{k n} \sigma_{k} F_{k}-2 \sum_{m=1}^{N-1} \rho_{m n} \sigma_{m} F_{m}\right) \sigma_{n} F_{n}+\sum_{p=1}^{N-1 N-1} \sum_{q=1} \rho_{p q} \sigma_{p} \sigma_{q} F_{p} F_{q}\right\} \frac{\partial^{2}}{\partial f_{n} \partial f_{k}} .
\end{gathered}
$$

The formal solution of Equation (36) is given by

$$
P\left(F_{1}, F_{2}, \cdots, F_{N}, \tau\right)=\mathrm{e}^{-r \tau} \exp \left\{\tau\left(\hat{L}_{N}+\hat{L}_{+}+\hat{L}_{N+}+\hat{L}_{R}\right)\right\} \times \max \left(F_{N}-F_{+}-K, 0\right) .
$$

Then we apply the Lie-Trotter operator splitting method [10] to obtain an approximation to the formal solution $P\left(F_{1}, F_{2}, \cdots, F_{N}, \tau\right)$, namely (see the Appendix)

$$
\begin{aligned}
& P^{L T}\left(F_{1}, F_{2}, \cdots, F_{N}, \tau\right) \\
& =\mathrm{e}^{-r \tau} \exp \left\{\tau\left(\hat{L}_{N}+\hat{L}_{+}+\hat{L}_{N+}\right)\right\} \exp \left\{\tau \hat{L}_{R}\right\} \max \left(F_{N}-F_{+}-K, 0\right) \\
& =\mathrm{e}^{-r \tau} \exp \left\{\tau\left(\hat{L}_{N}+\hat{L}_{+}+\hat{L}_{N+}\right)\right\} \max \left(F_{N}-F_{+}-K, 0\right),
\end{aligned}
$$

where the relation

$$
\hat{L}_{R} \max \left(F_{N}-F_{+}-K, 0\right)=0 \Rightarrow \exp \left\{\tau \hat{L}_{R}\right\} \max \left(F_{N}-F_{+}-K, 0\right)=\max \left(F_{N}-F_{+}-K, 0\right) .
$$

is utilized.

Next, in terms of the two new variables

$$
R_{1}=\frac{F_{N}}{F_{+}+K} \text { and } R_{2}=F_{+}+K \text {, }
$$

we rewrite $P^{L T}\left(F_{1}, F_{2}, \cdots, F_{N}, \tau\right)$ as follows: 


$$
P^{L T}\left(F_{1}, F_{2}, \cdots, F_{N}, \tau\right)=\mathrm{e}^{-r \tau} \exp \left\{\tau\left(\hat{L}_{A}+\hat{L}_{B}\right)\right\} R_{2} \max \left(R_{1}-1,0\right),
$$

where

$$
\begin{gathered}
\hat{L}_{A}=\frac{1}{2} \bar{\sigma}_{-}^{2} R_{1}^{2} \frac{\partial^{2}}{\partial R_{1}^{2}} \\
\hat{L}_{B}=\frac{1}{2} \bar{\sigma}_{+}^{2} R_{2}^{2} \frac{\partial^{2}}{\partial R_{2}^{2}}+\left(\tilde{\rho} \sigma_{N}-\bar{\sigma}_{+}\right) \bar{\sigma}_{+} R_{1} R_{2} \frac{\partial^{2}}{\partial R_{1} \partial R_{2}}-\left(\tilde{\rho} \sigma_{N}-\bar{\sigma}_{+}\right) \bar{\sigma}_{+} R_{1} \frac{\partial}{\partial R_{1}} \\
\bar{\sigma}_{-}=\sqrt{\sigma_{N}^{2}-2 \tilde{\rho} \sigma_{N} \bar{\sigma}_{+}+\bar{\sigma}_{+}^{2}} \\
\bar{\sigma}_{+}=\tilde{\sigma}_{+}\left(\frac{F_{+}}{F_{+}+K}\right) .
\end{gathered}
$$

It is clear that the exponential operator $\exp \left\{\tau\left(\hat{L}_{A}+\hat{L}_{B}\right)\right\}$ is difficult to evaluate, so we need to apply the Lie-Trotter operator splitting method [10] again to approximate the operator by $\exp \left\{\tau \hat{L}_{A}\right\} \exp \left\{\tau \hat{L}_{B}\right\}$. As a result, we obtain

$$
\begin{aligned}
P^{L T}\left(F_{1}, F_{2}, \cdots, F_{N}, \tau\right) & \approx \mathrm{e}^{-r \tau} \exp \left\{\tau \hat{L}_{A}\right\} \exp \left\{\tau \hat{L}_{B}\right\} R_{2} \max \left(R_{1}-1,0\right) \\
& \approx \mathrm{e}^{-r \tau} R_{2} \exp \left\{\tau \hat{L}_{A}\right\} \max \left(R_{1}-1,0\right)
\end{aligned}
$$

for

$$
\hat{L}_{B} R_{2} \max \left(R_{1}-1,0\right)=0 \Rightarrow \exp \left\{\tau \hat{L}_{B}\right\} R_{2} \max \left(R_{1}-1,0\right)=R_{2} \max \left(R_{1}-1,0\right) .
$$

Since $\bar{\sigma}_{\overline{-}}$ is independent of $R_{1}$, the standard workhorse of the Black-Scholes model can be used to evaluate $\exp \left\{\tau \hat{L}_{A}\right\} \max \left(R_{1}-1,0\right)$ such that

$$
P^{L T}\left(F_{1}, F_{2}, \cdots, F_{N}, \tau\right) \approx \mathrm{e}^{-r \tau} R_{2}\left\{R_{1} N\left(\theta_{1}\right)-N\left(\theta_{2}\right)\right\}
$$

where

$$
\begin{gathered}
\theta_{1}=\frac{1}{\bar{\sigma}_{-} \sqrt{\tau}}\left\{\ln \left(\frac{F_{N}}{F_{+}+K}\right)+\frac{1}{2} \bar{\sigma}_{-}^{2} \tau\right\} \\
\theta_{2}=\theta_{1}-\bar{\sigma}_{-} \sqrt{\tau} .
\end{gathered}
$$

It is obvious that this approximate solution is identical to the approximate price formula given in Equation (29). (Q.E.D.)

In terms of the spot asset prices, namely $S_{i} \equiv F_{i} \exp (-r \tau)$, the price formula given in Equation (29) becomes

$$
P_{\text {Kirk }}\left(S_{1}, S_{2}, \cdots, S_{N}, \tau\right)=S_{N} N\left(d_{1}\right)-\left(\sum_{j=1}^{N-1} S_{j}+K \mathrm{e}^{-r \tau}\right) N\left(d_{2}\right)
$$

where

$$
\begin{gathered}
d_{1}=\frac{\ln S_{N}-\ln \left(\sum_{j=1}^{N-1} S_{j}+K \mathrm{e}^{-r \tau}\right)}{\hat{\sigma}_{-} \sqrt{\tau}}+\frac{1}{2} \hat{\sigma}_{-} \sqrt{\tau} \\
d_{2}=d_{1}-\hat{\sigma}_{-} \sqrt{\tau} \\
\hat{\sigma}_{-}=\sqrt{\sigma_{N}^{2}-2 \bar{\rho} \sigma_{N} \hat{\sigma}_{+}+\hat{\sigma}_{+}^{2}} \\
\hat{\sigma}_{+}=\frac{\sqrt{\sum_{j, k=1}^{N-1} \rho_{j k} \sigma_{j} \sigma_{k} S_{j} S_{k}}}{\sum_{j=1}^{N-1} S_{j}+K \mathrm{e}^{-r \tau}}
\end{gathered}
$$




$$
\bar{\rho}=\frac{1}{\hat{\sigma}_{+}}\left(\frac{\sum_{j=1}^{N-1} \rho_{j N} \sigma_{j} S_{j}}{\sum_{j=1}^{N-1} S_{j}}\right) .
$$

Obviously, this approximate price formula resembles the price formula of Kirk's approximation in the two-asset case very closely. In fact, by setting $N=2$ we can recover Kirk's approximation readily. Moreover, for the Lie-Trotter splitting approximation to be valid, we need to require $\hat{\sigma}_{-}^{2} \tau$ to be sufficiently small, namely $\bar{\sigma}_{-}^{2} \tau \ll 1$. In accordance with Equation (58), this implies that the extended Kirk approximation is more favourable for those cases with positive effective correlation $\bar{\rho}$.

\section{Illustrative Numerical Examples for the Three-Asset Spread Options}

In this section, illustrative numerical examples are presented to demonstrate the accuracy of the extended Kirk approximation for the three-asset spread options. Although most spread options involve two assets only, yet there is a growing demand for three-asset spread options which can be found in the models for power plants or their financial equivalents - tolling contracts. We examine a simple three-asset spread option with the final payoff $\max \left(S_{3}-S_{1}-S_{2}-K, 0\right)$. Table 1 tabulates the approximate option prices estimated by the extended Kirk approximation for different values of the strike price $K$ and time-to-maturity $T$. Other input model parameters are set as follows: $r=0.05, \sigma_{1}=\sigma_{2}=\sigma_{3}=0.3, \rho_{12}=0.4, \rho_{23}=0.2, \rho_{13}=0.8, S_{1}=50$, $S_{2}=60$ and $S_{3}=150$. Monte Carlo estimates and the corresponding standard deviations are also presented for comparison. It is observed that the computed errors of the approximate option prices are capped at $1 \%$ (in magnitude). In fact, most of them are less than $0.5 \%$. Then, in Table 2 the effect of increasing the three volatilities (from 0.3 to 0.6 ) upon the approximate estimation of the option prices is investigated. Obviously only a slight increase occurs in the computed errors, and these errors are still less than $1 \%$ (in magnitude). Finally, we study a case in which all the three volatilities are different, namely $\sigma_{1}=0.5, \sigma_{2}=0.4$ and $\sigma_{3}=0.3$, while the other parameters remain the same. According to Table 3, the computed errors generally increase a little bit in this case but they do not exceed 1.3\% (in magnitude). As a result, it can be concluded that the extended Kirk approximation for the three-asset spread option is found to be very accurate and efficient.

\section{Conclusion}

In this paper, we have proved that Kirk's approximation for two-asset spread options can be rigorously derived

Table 1. Prices of a European three-asset call spread option. Other input parameters are: $\mathrm{r}=0.05, \sigma_{1}=\sigma_{2}=\sigma_{3}=0.3, \rho_{12}=$ 0.4, $\rho_{23}=0.2, \rho_{13}=0.8, \mathrm{~S}_{1}=50, \mathrm{~S}_{2}=60$ and $\mathrm{S}_{3}=150$. Here "EK" refers to the extended Kirk approximation while "MC" denotes the Monte Carlo estimates with 900,000,000 replications. The relative errors of the "EK" option prices with respect to the "MC" estimates are also presented.

\begin{tabular}{|c|c|c|c|c|c|}
\hline $\mathrm{K} \backslash \mathrm{T}$ & 0.25 & 0.5 & 1 & 2 & \\
\hline \multirow[t]{3}{*}{30} & 13.5410 & 16.4210 & 20.7761 & 27.1974 & EK \\
\hline & $-0.3 \%$ & $-0.3 \%$ & $-0.3 \%$ & $-0.3 \%$ & error \\
\hline & $13.5763 \pm 0.0089$ & $16.4735 \pm 0.0142$ & $20.8471 \pm 0.0185$ & $27.2841 \pm 0.0264$ & $\mathrm{MC}$ \\
\hline \multirow[t]{3}{*}{35} & 10.3383 & 13.5024 & 18.1191 & 24.8196 & EK \\
\hline & $-0.2 \%$ & $-0.2 \%$ & $-0.2 \%$ & $-0.2 \%$ & error \\
\hline & $10.3576 \pm 0.0086$ & $13.5286 \pm 0.0124$ & $18.1541 \pm 0.0176$ & $24.8573 \pm 0.0276$ & $\mathrm{MC}$ \\
\hline \multirow[t]{3}{*}{40} & 7.6613 & 10.9586 & 15.7231 & 22.6176 & EK \\
\hline & $0.0 \%$ & $0.0 \%$ & $0.0 \%$ & $0.1 \%$ & error \\
\hline & $7.6608 \pm 0.0068$ & $10.9572 \pm 0.0109$ & $15.7197 \pm 0.0161$ & $22.6072 \pm 0.0244$ & $\mathrm{MC}$ \\
\hline \multirow[t]{3}{*}{45} & 5.5097 & 8.7824 & 13.5805 & 20.5856 & EK \\
\hline & $0.4 \%$ & $0.3 \%$ & $0.3 \%$ & $0.3 \%$ & error \\
\hline & $5.4903 \pm 0.0051$ & $8.7565 \pm 0.0106$ & $13.5421 \pm 0.0141$ & $20.5302 \pm 0.0260$ & MC \\
\hline \multirow[t]{3}{*}{50} & 3.8470 & 6.9540 & 11.6795 & 18.7162 & EK \\
\hline & $0.9 \%$ & $0.7 \%$ & $0.6 \%$ & $0.5 \%$ & error \\
\hline & $3.8140 \pm 0.0040$ & $6.9058 \pm 0.0086$ & $11.6109 \pm 0.0136$ & $18.6195 \pm 0.0245$ & MC \\
\hline
\end{tabular}


Table 2. Prices of a European three-asset call spread option. Other input parameters are: $\mathrm{r}=0.05, \sigma_{1}=\sigma_{2}=\sigma_{3}=0.6, \rho_{12}=$ $0.4, \rho_{23}=0.2, \rho_{13}=0.8, \mathrm{~S}_{1}=50, \mathrm{~S}_{2}=60$ and $\mathrm{S}_{3}=150$. Here "EK" refers to the extended Kirk approximation while "MC" denotes the Monte Carlo estimates with 900,000,000 replications. The relative errors of the "EK" option prices with respect to the "MC" estimates are also presented.

\begin{tabular}{|c|c|c|c|c|c|}
\hline $\mathrm{K} \backslash \mathrm{T}$ & 0.25 & 0.5 & 1 & 2 & \\
\hline \multirow[t]{3}{*}{30} & 20.1436 & 26.0640 & 34.5186 & 46.3820 & EK \\
\hline & $-0.3 \%$ & $-0.3 \%$ & $-0.1 \%$ & $0.3 \%$ & error \\
\hline & $20.2066 \pm 0.0168$ & $26.1269 \pm 0.0278$ & $34.5402 \pm 0.0425$ & $46.2242 \pm 0.0716$ & MC \\
\hline \multirow[t]{3}{*}{35} & 17.4529 & 23.5976 & 32.2944 & 44.4495 & EK \\
\hline & $-0.1 \%$ & $0.0 \%$ & $0.1 \%$ & $0.5 \%$ & error \\
\hline & $17.4778 \pm 0.0172$ & $23.6076 \pm 0.0268$ & $32.2508 \pm 0.0390$ & $44.2275 \pm 0.0787$ & MC \\
\hline \multirow[t]{3}{*}{40} & 15.0417 & 21.3320 & 30.2150 & 42.6221 & EK \\
\hline & $0.1 \%$ & $0.2 \%$ & $0.4 \%$ & $0.7 \%$ & error \\
\hline & $15.0290 \pm 0.0171$ & $21.2938 \pm 0.0266$ & $30.1079 \pm 0.0362$ & $42.3425 \pm 0.0656$ & MC \\
\hline \multirow[t]{3}{*}{45} & 12.9002 & 19.2587 & 28.2733 & 40.8938 & EK \\
\hline & $0.4 \%$ & $0.4 \%$ & $0.6 \%$ & $0.9 \%$ & error \\
\hline & $12.8527 \pm 0.0182$ & $19.1753 \pm 0.0234$ & $28.1113 \pm 0.0381$ & $40.5466 \pm 0.0628$ & MC \\
\hline \multirow[t]{3}{*}{50} & 11.0139 & 17.3676 & 26.4620 & 39.2590 & EK \\
\hline & $0.7 \%$ & $0.7 \%$ & $0.8 \%$ & $1.0 \%$ & error \\
\hline & $10.9347 \pm 0.0151$ & $17.2410 \pm 0.0236$ & $26.2513 \pm 0.0345$ & $38.8658 \pm 0.0637$ & $\mathrm{MC}$ \\
\hline
\end{tabular}

Table 3. Prices of a European three-asset call spread option. Other input parameters are: $\mathrm{r}=0.05, \sigma_{1}=0.5, \sigma_{2}=0.4, \sigma_{3}=0.3$, $\rho_{12}=0.4, \rho_{23}=0.2, \rho_{13}=0.8, \mathrm{~S}_{1}=50, \mathrm{~S}_{2}=60$ and $\mathrm{S}_{3}=150$. Here "EK" refers to the extended Kirk approximation while "MC" denotes the Monte Carlo estimates with 900,000,000 replications. The relative errors of the "EK" option prices with respect to the "MC" estimates are also presented.

\begin{tabular}{|c|c|c|c|c|c|}
\hline $\mathrm{K} \backslash \mathrm{T}$ & 0.25 & 0.5 & 1 & 2 & \\
\hline \multirow[t]{3}{*}{30} & 13.8987 & 16.9731 & 21.6091 & 28.4620 & EK \\
\hline & $-0.5 \%$ & $-0.6 \%$ & $-0.8 \%$ & $-1.2 \%$ & error \\
\hline & $13.9635 \pm 0.0092$ & $17.0801 \pm 0.0119$ & $21.7922 \pm 0.0191$ & $28.8014 \pm 0.0242$ & MC \\
\hline \multirow[t]{3}{*}{35} & 10.6503 & 13.9613 & 18.8011 & 25.8630 & EK \\
\hline & $-0.4 \%$ & $-0.5 \%$ & $-0.7 \%$ & $-1.1 \%$ & error \\
\hline & $10.6905 \pm 0.0078$ & $14.0301 \pm 0.0118$ & $18.9316 \pm 0.0163$ & $26.1380 \pm 0.0238$ & MC \\
\hline \multirow[t]{3}{*}{40} & 7.9011 & 11.3085 & 16.2480 & 23.4424 & EK \\
\hline & $-0.1 \%$ & $-0.2 \%$ & $-0.4 \%$ & $-0.9 \%$ & error \\
\hline & $7.9101 \pm 0.0074$ & $11.3342 \pm 0.0104$ & $16.3206 \pm 0.0172$ & $23.6452 \pm 0.0223$ & $\mathrm{MC}$ \\
\hline \multirow[t]{3}{*}{45} & 5.6664 & 9.0191 & 13.9501 & 21.1989 & EK \\
\hline & $0.4 \%$ & $0.2 \%$ & $-0.1 \%$ & $-0.6 \%$ & error \\
\hline & $5.6426 \pm 0.0064$ & $9.0004 \pm 0.0092$ & $13.9627 \pm 0.0145$ & $21.3286 \pm 0.0210$ & MC \\
\hline \multirow[t]{3}{*}{50} & 3.9253 & 7.0838 & 11.9023 & 19.1291 & EK \\
\hline & $1.3 \%$ & $0.8 \%$ & $0.4 \%$ & $-0.3 \%$ & error \\
\hline & $3.8758 \pm 0.0058$ & $7.0242 \pm 0.0081$ & $11.8587 \pm 0.0147$ & $19.1882 \pm 0.0216$ & MC \\
\hline
\end{tabular}

by applying the Lie-Trotter operator splitting approximation to the Black-Scholes equation, and that for cases with vanishing strike prices, the Lie-Trotter splitting approximation becomes exact and Margrabe's formula is recovered. Our derivation also shows that Kirk's approximation is more favourable for those cases with two positively correlated assets. Moreover, we apply the Lie-Trotter operator splitting method to obtain a generalisation of Kirk's approximation for multi-asset spread options in a straightforward manner. The derived price formula for the multi-asset spread option closely resembles Kirk's approximation in the two-asset case. By setting the total number of assets to be two, we can recover Kirk's approximation readily. Thus, the generalisation possesses the same nice features as Kirk’s approximation. For instance, as shown by the illustrative 
examples, the generalization is found to be very accurate and efficient in pricing the multi-asset spread options. All in all, our approach is able to provide a new perspective on Kirk's approximation and the generalisation; that is, they are simply equivalent to the Lie-Trotter operator splitting approximation to the Black-Scholes equation.

\section{References}

[1] Carmona, R. and Durrleman, V. (2003) Pricing and Hedging Spread Options. SIAM Review, 45, 627-685. http://dx.doi.org/10.1137/S0036144503424798

[2] Deng, S.J., Li, M. and Zhou, J. (2008) Closed-Form Approximation for Spread Option Prices and Greeks. Journal of Derivatives, 15, 58-80. http://dx.doi.org/10.3905/jod.2008.702506

[3] Bjerksund, P. and Stensland, G. (2011) Closed Form Spread Option Valuation. Quantitative Finance, iFirst, 1-10.

[4] Venkatramana, A. and Alexander, C. (2011) Closed form Approximation for Spread Options. Applied Mathematical Finance, 18, 447-472. http://dx.doi.org/10.1080/1350486X.2011.567120

[5] Kirk, E. (1995) Correlation in the Energy Markets. Managing Energy Price Risk. Risk Publications and Enron, London, 71-78.

[6] Margrabe, W. (1978) The Value of an Option to Exchange One Asset for Another. Journal of Finance, 33, 177-186. http://dx.doi.org/10.1111/j.1540-6261.1978.tb03397.x

[7] Lo, C.F. (2013) A Simple Derivation of Kirk’s Approximation for Spread Options. Applied Mathematics Letters, 26, 904-907. http://dx.doi.org/10.1016/j.aml.2013.04.004

[8] Trotter, H.F. (1958) Approximation of Semi-Groups of Operators. Pacific Journal of Mathematics, 8, 887-919. http://dx.doi.org/10.2140/pjm.1958.8.887

[9] Li, M., Zhou, J. and Deng, S.J. (2010) Multi-Asset Spread Option Pricing and Hedging. Quantitative Finance, 10, 305-324. http://dx.doi.org/10.1080/14697680802626323

[10] Trotter, H.F. (1959) On the Product of Semi-Groups of Operators. Proceedings of the American Mathematical Society, 10, 545-551. http://dx.doi.org/10.1090/S0002-9939-1959-0108732-6

[11] Suzuki, M. (1985) Decomposition Formulas of Exponential Operators and Lie Exponentials with Some Applications to Quantum Mechanics and Statistical Physics. Journal of Mathematical Physics, 26, 601-612. http://dx.doi.org/10.1063/1.526596

[12] Drozdov, A.N. and Brey, J.J. (1998) Operator Expansions in Stochastic Dynamics. Physical Review E, 57, $1284-1289$. http://dx.doi.org/10.1103/PhysRevE.57.1284

[13] Hatano, N. and Suzuki, M. (2005) Finding Exponential Product Formulas of Higher Orders. Lecture Notes in Physics, 679, 37-68.

[14] Blanes, S., Casas, F., Chartier, P. and Murua, A. (2013) Optimized Higher-Order Splitting Methods for Some Classes of Parabolic Equations. Mathematics of Computation, 82, 1559-1576. http://dx.doi.org/10.1090/S0025-5718-2012-02657-3 


\section{Appendix: Lie-Trotter Operator Splitting Method}

Suppose that one needs to exponentiate an operator $\hat{C}$ which can be split into two different parts, namely $\hat{A}$ and $\hat{B}$. For simplicity, let us assume that $\hat{C}=\hat{A}+\hat{B}$, where the exponential operator $\exp (\hat{C})$ is difficult to evaluate but $\exp (\hat{A})$ and $\exp (\hat{B})$ are either solvable or easy to deal with. Under such circumstances, the exponential operator $\exp (\varepsilon \hat{C})$, with $\varepsilon$ being a small parameter, can be approximated by the Lie-Trotter operator splitting formula:

$$
\exp (\varepsilon \hat{C})=\exp (\varepsilon \hat{A}) \exp (\varepsilon \hat{B})+O\left(\varepsilon^{2}\right) .
$$

This can be seen as the approximation to the solution at $t=\varepsilon$ of the equation $\mathrm{d} \hat{Y} / \mathrm{d} t=(\hat{A}+\hat{B}) \hat{Y}$ by a composition of the exact solutions of the equations $\mathrm{d} \hat{Y} / \mathrm{d} t=\hat{A} \hat{Y}$ and $\mathrm{d} \hat{Y} / \mathrm{d} t=\hat{B} \hat{Y}$ at time $t=\varepsilon$. Details of the Lie-Trotter splitting approximation can be found in Trotter [8] [10], Suzuki [11], Drozdov and Brey [12], Hatano and Suzuki [13], and Blanes et al. [14]. The Lie-Trotter splitting approximation is particularly useful for studying the short-time behaviour of the solutions of evolutionary partial differential equations of parabolic type because for this class of problems it is sensible to split the spatial differential operator into several parts each of which corresponds to a different physical contribution (e.g., reaction and diffusion). 